\title{
Inactivation of focal adhesion kinase in cardiomyocytes promotes eccentric cardiac hypertrophy and fibrosis in mice
}

\author{
Xu Peng, ${ }^{1}$ Marc S. Kraus, ${ }^{2}$ Huijun Wei, ${ }^{1}$ Tang-Long Shen, ${ }^{1}$ Romain Pariaut, ${ }^{2}$ Ana Alcaraz, ${ }^{3}$ \\ Guangju Ji, ${ }^{3}$ Lihong Cheng, ${ }^{4}$ Qinglin Yang, ${ }^{4}$ Michael I. Kotlikoff, ${ }^{3}$ Ju Chen, ${ }^{5}$ \\ Kenneth Chien, ${ }^{5}$ Hua Gu, 6,7 and Jun-Lin Guan ${ }^{1}$ \\ 1Department of Molecular Medicine, ${ }^{2}$ Department of Clinical Sciences, and ${ }^{3}$ Department of Biomedical Sciences, College of Veterinary Medicine, \\ Cornell University, Ithaca, New York, USA. ${ }^{4}$ Cardiovascular Research Institute, Morehouse School of Medicine, Atlanta, Georgia, USA. \\ ${ }^{5}$ Department of Medicine, University of California at San Diego School of Medicine, La Jolla, California, USA. ${ }^{6}$ Laboratory of Immunology, \\ National Institute of Allergy and Infectious Diseases, NIH, Rockville, Maryland, USA. ${ }^{7}$ Department of Microbiology, \\ Columbia University College of Physicians and Surgeons, New York, New York, USA.
}

\begin{abstract}
Focal adhesion kinase (FAK) is a cytoplasmic tyrosine kinase that plays a major role in integrin signaling pathways. Although cardiovascular defects were observed in FAK total KO mice, the embryonic lethality prevented investigation of FAK function in the hearts of adult animals. To circumvent these problems, we created mice in which FAK is selectively inactivated in cardiomyocytes (CFKO mice). We found that CFKO mice develop eccentric cardiac hypertrophy (normal LV wall thickness and increased left chamber dimension) upon stimulation with angiotensin II or pressure overload by transverse aortic constriction as measured by echocardiography. We also found increased heart/body weight ratios, elevated markers of cardiac hypertrophy, multifocal interstitial fibrosis, and increased collagen I and VI expression in CFKO mice compared with control littermates. Spontaneous cardiac chamber dilation and increased expression of hypertrophy markers were found in the older CFKO mice. Analysis of cardiomyocytes isolated from CFKO mice showed increased length but not width. The myocardium of CFKO mice exhibited disorganized myofibrils with increased nonmyofibrillar space filled with swelled mitochondria. Last, decreased tyrosine phosphorylation of FAK substrates p130Cas and paxillin were observed in CFKO mice compared with the control littermates. Together, these results provide strong evidence for a role of FAK in the regulation of heart hypertrophy in vivo.
\end{abstract}

\section{Introduction}

Cardiac hypertrophy is an adaptation process in response to sustained hemodynamic overload in the fully developed adult heart, and prolongation of this process leads to arrhythmia, congestive heart failure, and sudden death. Increased diastolic stress or changes in cytoskeletal structures can induce eccentric hypertrophy and are associated with an especially poor prognosis. Understanding the mechanisms involved in eccentric cardiac hypertrophy could provide insights into the molecular basis for pathogenesis of adult heart disease. Furthermore, this may provide novel therapeutic insights in the future (1-6).

Various humoral factors, such as vasoactive peptides, catecholamines, cytokines, and growth factors, can contribute to the development of cardiac hypertrophy, but mechanical stress is considered the primary trigger to induce hypertrophy in the overloaded myocardium $(7,8)$. Integrins, which play a role in linking the force-generating actin cytoskeleton to the extracellular matrix, are considered to be potential receptors for mechanical stress (8). Magnetic beads coated with an integrin ligand are capable of transferring mechanical stress to the cytoskeleton (9). Indeed, a

Nonstandard abbreviations used: ANP, atrial natriuretic peptide; BNP, brain natriuretic peptide; FAK, focal adhesion kinase; $\beta$-MHC, myosin heavy chain $\beta ;$ MLC2v, myosin light chain $2 \mathrm{v}$; NRCM, neonatal rat cardiomyocyte; SK-actin, $\alpha$-skeletal actin; TAC, transverse aortic constriction; WGA, wheat germ agglutinin.

Conflict of interest: The authors have declared that no conflict of interest exists.

Citation for this article: J. Clin. Invest. 116:217-227 (2006)

doi:10.1172/JCI24497. role of integrins in heart development and hypertrophy has been supported by a number of previous studies. Integrin $\beta 1-\mathrm{KO}$ mice die in utero by E5.5 due to gastrulation defects $(10,11)$. Interestingly, chimeric mice generated from integrin $\beta 1$-null ES cells showed delayed development and differentiation of cardiac lineage, as well as abnormal sarcomerogenesis of these cardiac-like cells. Conditional deletion of integrin $\beta 1$ in ventricular cardiomyocytes resulted in myocardial fibrosis and cardiac chamber dilation (12). These and other in vitro results suggested that integrins and their downstream signaling pathways play important roles in the regulation of heart development and hypertrophy.

Focal adhesion kinase (FAK) is a cytoplasmic tyrosine kinase that plays a major role in integrin-mediated signal transduction (13-17). Recent studies have suggested that FAK is important in transducing mechanical stimuli to downstream biochemical pathways that lead to cardiomyocyte hypertrophy. FAK activation occurs in isolated cardiomyocytes subjected to pulsatile mechanical stretch and in overloaded feline and rat myocardium (18-21). Recent data also indicate that increases in LV pressure are accompanied by an increase in tyrosine phosphorylation of FAK, as well as an increased association of FAK with Src and Grb2 (22). Aside from its well-established role in mediating integrin signaling, FAK may also participate in signal transduction by $G$ protein-coupled receptors. Several bioactive peptides, including endothelin, bombesin, and Ang II, which regulate heart function, activate FAK via their specific cell-surface $\mathrm{G}$ protein-coupled receptors (23). In addition, VEGF can induce activation and subcellular translocation of FAK 
A

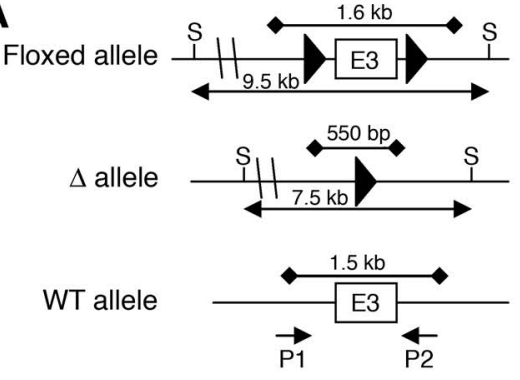

B

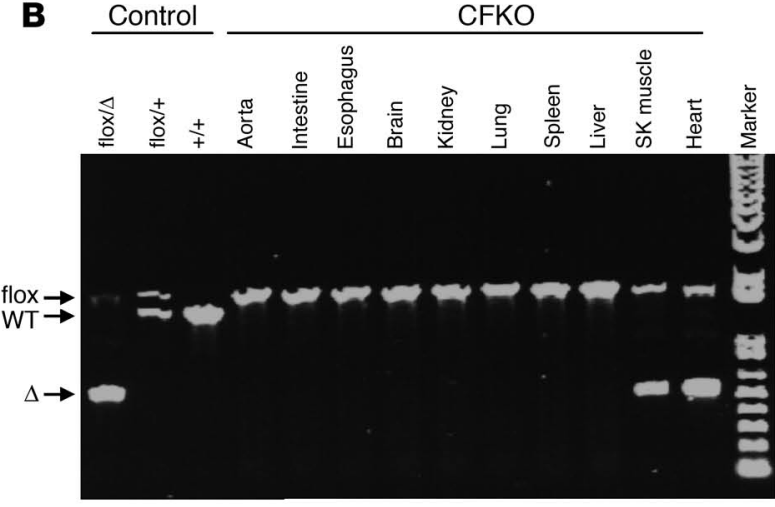

C

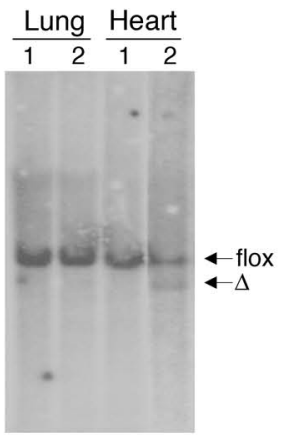

D

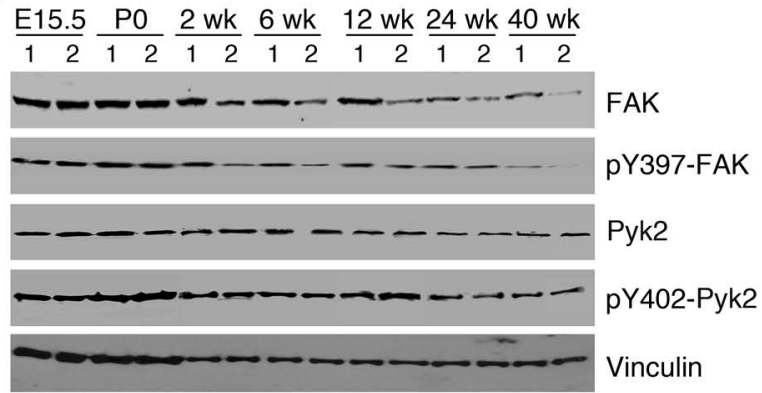

E

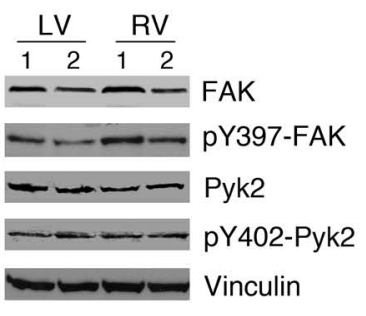

$\mathbf{F}$

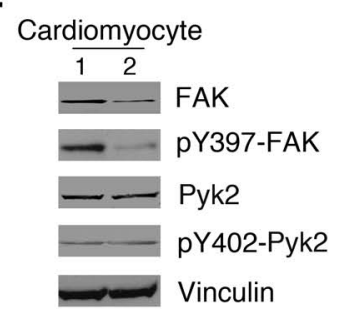

Figure 1

Ventricular cardiomyocyte-specific deletion of FAK. (A) Schematics of FAK WT, floxed, and deleted $(\Delta)$ alleles. Large filled triangles represent loxP sites. Horizontal lines with arrows indicate the expected sizes of DNA bands in Southern blotting of the floxed and $\Delta$ alleles. Horizontal lines with filled diamonds indicate the expected sizes of DNA bands in PCR. The location of the Sacl (S) restriction sites and the primers P1 and P2 (arrows) are shown. E3, exon 3. (B) Genomic DNA from different tissues of CFKO mice were amplified by PCR with DNA from tails of various control mice (3 left lanes) as controls. The DNA bands corresponding to the flox, WT, and $\Delta$ alleles are marked on the left. SK, skeletal muscle. (C) Southern blotting analysis of the genomic DNA from lungs and ventricles of control (lane 1) and CFKO (lane 2) mice after Sacl digestion. The positions of floxed and $\Delta$ alleles are marked on the right. (D) Lysates were prepared from ventricles of control and CFKO mice at different time points, as indicated. They were then analyzed by Western blotting using various antibodies, as indicated. (E and F) Lysates were prepared from LV, RV, or isolated cardiomyocytes from control and CFKO mice, as indicated. They were then analyzed by Western blotting using various antibodies, as indicated.

from perinuclear sites to focal adhesions in cultured neonatal cardiomyocytes (24). Taken together, these data suggest that FAK may function as a converging point in the signaling pathways triggered by integrin, $G$ protein-coupled, and growth factor receptors that are important in the regulation of cardiomyocyte function.

Consistent with its critical roles in vitro, FAK gene inactivation in mice resulted in a lethal embryonic phenotype with major defects in the axial mesoderm and cardiovascular system $(25,26)$. Neither a normal heart nor fully developed blood vessels were present in the FAK-null embryos. The analysis of FAK total KO embryos as well as studies using various in vitro systems suggested a potential role of FAK in heart development and function. However, the lethal embryonic phenotype of FAK-null mice precluded further studies of the role of FAK in heart development and function in adults. To overcome this, we generated a cardiac ventricle-restricted FAK-KO mouse. We found that specific inactivation of FAK in ventricular cardiomyocytes promoted eccentric cardiac hypertrophy and fibrosis in response to Ang II stimulation. We also showed that these conditional FAK-KO mice developed spontaneous left-ventricular chamber dilation by 9 months of age. These findings suggest that FAK is an important regulator of heart hypertrophy in vivo.

\section{Results}

Generation of ventricular cardiomyocyte-specific FAK-null mice. To generate mice with ventricular cardiomyocyte-specific KO of the FAK gene, we employed a Cre/loxP strategy by intercrossing mice containing a floxed FAK allele (27) with MLC2vCreKI mice (28). Floxed FAK mice were generated recently in our laboratory and contain 2 loxP sites flanking exon 3 of the FAK gene. Cre-mediated deletion of exon 3 leads to a frameshift mutation because of direct splicing from exon 2 (containing the ATG initiation codon) to exon 4 , which produces a small truncated and nonfunctional peptide $(\sim 70$ amino acids) lacking the majority of FAK sequences, including the kinase domain and the focal adhesion-targeting sequence. MLC2vCreKI mice were created by inserting Cre recombinase into the myosin light chain $2 \mathrm{v}$ (MLC2v) gene locus, and they have been shown to mediate recombinations between loxP sequences to cleave floxed genes effectively and specifically in ventricular cardiomyocytes, when crossed with mice containing floxed alleles $(12,28-34)$. Matings between $F A K^{\text {flox } / \text { flox }} M L C 2 v^{+/+}$and $F A K^{\text {flox/+ }}$ $M L C 2 v C r e^{K I /+}$ mice yielded $F A K^{\text {flox } /+} M L C 2 v^{+/+}, F A K^{\text {flox } / \text { flox } M L C 2 v^{+/+}}$ (designated as control), FAK flox/+$M L C 2 v \mathrm{Cre}^{\mathrm{KI} /+}$, and $F A K^{f l o x} / f l o x$ $M L C 2 v \mathrm{Cr}^{K I /+}$ (conditional FAK KO, designated as CFKO) pups at the expected 1:1:1:1 Mendelian ratio. 
Table 1

Echocardiographic parameters in anesthetized mice

\begin{tabular}{|c|c|c|c|c|c|c|c|c|c|}
\hline & $n$ & Heart rate (bpm) & IVSd (cm) & IVSs (cm) & LVIDd (cm) & LVIDs (cm) & LVFWd (cm) & LVFWs (cm) & FS (\%) \\
\hline \multicolumn{10}{|l|}{ 12-Week-old } \\
\hline Flox control & 4 & $289 \pm 22$ & $0.08 \pm 0.01$ & $0.12 \pm 0.02$ & $0.30 \pm 0.04$ & $0.18 \pm 0.04$ & $0.09 \pm 0.03$ & $0.13 \pm 0.02$ & $43.7 \pm 0.12$ \\
\hline Cre control & 4 & $295 \pm 100$ & $0.07 \pm 0.01$ & $0.11 \pm 0.01$ & $0.29 \pm 0.03$ & $0.19 \pm 0.02$ & $0.11 \pm 0.01$ & $0.14 \pm 0.01$ & $34.4 \pm 0.2$ \\
\hline CFKO & 4 & $298 \pm 74.5$ & $0.08 \pm 0.01$ & $0.12 \pm 0.01$ & $0.30 \pm 0.03$ & $0.18 \pm 0.04$ & $0.10 \pm 0.02$ & $0.13 \pm 0.02$ & $40.5 \pm 10$ \\
\hline \multicolumn{10}{|c|}{ 12-Week-old with Ang II stimulation } \\
\hline Flox control & 9 & $299 \pm 31.3$ & $0.09 \pm 0.02$ & $0.12 \pm 0.02$ & $0.30 \pm 0.02$ & $0.20 \pm 0.03$ & $0.10 \pm 0.01$ & $0.14 \pm 0.03$ & $32.1 \pm 10$ \\
\hline Cre control & 9 & $267 \pm 55.6$ & $0.10 \pm 0.02$ & $0.13 \pm 0.03$ & $0.32 \pm 0.03$ & $0.19 \pm 0.04$ & $0.11 \pm 0.03$ & $0.15 \pm 0.02$ & $39.3 \pm 13.2$ \\
\hline CFKO & 8 & $265 \pm 59.3$ & $0.09 \pm 0.01$ & $0.12 \pm 0.01$ & $0.35 \pm 0.02^{\mathrm{A}}$ & $0.25 \pm 0.02^{A}$ & $0.09 \pm 0.01$ & $0.13 \pm 0.02$ & $29.8 \pm 5.7$ \\
\hline \multicolumn{10}{|c|}{ 10-Week-old with TAC surgery } \\
\hline Flox control & 7 & $345 \pm 23$ & $0.08 \pm 0.01$ & $0.11 \pm 0.01$ & $0.30 \pm 0.04$ & $0.21 \pm 0.05$ & $0.09 \pm 0.02$ & $0.11 \pm 0.02$ & $31 \pm 10$ \\
\hline Cre control & 6 & $301 \pm 131$ & $0.08 \pm 0.01$ & $0.11 \pm 0.01$ & $0.31 \pm 0.03$ & $0.22 \pm 0.02$ & $0.09 \pm 0.01$ & $0.11 \pm 0.02$ & $30 \pm 5.8$ \\
\hline CFKO & 7 & $300 \pm 68$ & $0.09 \pm 0.01$ & $0.11 \pm 0.01$ & $0.36 \pm 0.03^{A}$ & $0.27 \pm 0.04^{A}$ & $0.09 \pm 0.02$ & $0.12 \pm 0.02$ & $33 \pm 12$ \\
\hline \multicolumn{10}{|l|}{ 9-Month-old } \\
\hline Flox control & 6 & $286 \pm 62.1$ & $0.09 \pm 0.01$ & $0.13 \pm 0.02$ & $0.32 \pm 0.03$ & $019 \pm 0.04$ & $0.09 \pm 0.02$ & $0.13 \pm 0.03$ & $40.9 \pm 14.9$ \\
\hline Cre control & 6 & $335 \pm 107$ & $0.09 \pm 0.02$ & $0.14 \pm 0.02$ & $0.30 \pm 0.02$ & $0.19 \pm 0.05$ & $0.10 \pm 0.02$ & $0.14 \pm 0.03$ & $40.7 \pm 14.2$ \\
\hline CFKO & 7 & $294 \pm 85.2$ & $0.08 \pm 0.01$ & $0.12 \pm 0.01$ & $0.37 \pm 0.04^{\mathrm{A}}$ & $0.27 \pm 0.03^{\mathrm{A}}$ & $0.09 \pm 0.01$ & $0.12 \pm 0.01$ & $27.9 \pm 6.2$ \\
\hline
\end{tabular}

Values were quantified from 3 separate M-mode measurements. ${ }^{A} P<0.05$, compared with Cre and flox control mice. IVSd, interventricular septal thickness at end diastole; IVSs, interventricular septal thickness at end systole; LVIDd, LV internal diameter at end diastole; LVIDs, LV internal diameter at end systole; LVFWd, LV free wall thickness at end diastole; LVFWs, LV free wall thickness at end systole; FS, fractional shortening.

To evaluate specificity of FAK deletion in CFKO mice, genomic DNA were extracted from heart and other tissues of the CFKO mice and subjected to PCR analysis to detect different FAK alleles (see Figure 1A). Consistent with the expression patterns of the endogenous MLC2v gene $(28,35)$, the recombined FAK-deleted allele was detected only in heart and skeletal muscle, but not other tissues, including aorta, intestine, esophagus, brain, kidney, lung, spleen, and liver (Figure 1B). Genomic DNA from the heart and lungs of CFKO and control littermate mice were also examined by Southern blotting to quantify the efficiency of FAK gene deletion. Figure 1C shows the FAK deletion allele in DNA extracted from the heart of CFKO, but not the control littermate, mice. DNA from lungs of both CFKO and control mice contained only the floxed alleles, further confirming the specificity as suggested by PCR studies (see Figure 1B). Densitometric analysis of the FAKdeleted versus -floxed alleles in the heart samples of CFKO mice from multiple experiments suggested that approximately $20 \%$ of the FAK-floxed allele in the heart have undergone recombination in CFKO mice. Previous studies have shown that about $14 \%$ of adult mouse ventricular cells are cardiomyocytes, and more than $90 \%$ of cardiomyocytes are binucleated (36). Thus, the $20 \%$ recombination of the FAK-floxed allele observed in the whole heart of CFKO mice suggests a FAK recombination efficiency of approximately $80 \%$ in cardiomyocytes.

To evaluate reduction of FAK protein expression, lysates were prepared from cardiac ventricles of CFKO and control mice at embryonic stage E15.5, newborn (postnatal day 0), and 2 weeks to 40 weeks of age, and the lysates were analyzed by Western blot analysis (Figure 1D). Although MLC2vCreKI mice have been shown to mediate recombination of floxed genes as early as E8.5 (28), FAK expression was similar for CFKO and control mice at E15.5 and postnatal day 0. A significant reduction in FAK protein level was observed for the CFKO mice (lane 2) compared with the control littermates (lane 1) in hearts from 2 weeks till 40 weeks after birth. Consistent with gene analysis results, FAK expression in the lungs was similar in CFKO and control mice (data not shown). Reduction of FAK expression was observed in both LVs and RVs in CFKO mice compared with control littermates (Figure 1E). As expected, a significant decrease in FAK expression was also found in isolated cardiomyocytes from CFKO mice compared with control mice (Figure 1F). Furthermore, FAK phosphorylation at $\mathrm{Y} 397$ was reduced in proportion to its reduced protein level in the heart as well as isolated cardiomyocytes from CFKO mice (Figure 1, D-F, second panels). Immunoblotting for Pyk2 and phosphorylated Pyk2 (at Y402) showed similar expression levels and activation state of Pyk2 in CFKO and control mice (third and fourth panels). Similar loading in all lanes was verified by Western blotting with anti-vinculin, as shown in the bottom panels. Together, these results suggested specific and efficient deletion of the FAK gene in cardiomyocytes of the CFKO mice, consistent with several previous reports using MLC2vCreKI mice $(12,28-34)$. These data also suggest that FAK deletion did not result in a compensatory increase in the activation of the residual FAK or the expression or activity of the related kinase Pyk2.

Inactivation of FAK does not affect cardiac functions. In contrast to mice with total FAK KO, which have cardiac defects in early embryogenesis, CFKO mice have normal embryonic viability. Pups were born at the expected Mendelian frequency and grew to adulthood. CFKO mice were externally indistinguishable from their control littermates. To investigate whether the cardiacspecific deletion of FAK would affect cardiac morphology and function in vivo, echocardiography and cardiac catheterization were performed on the CFKO and control mice. Examination of cardiac dimensions and fractional shortening percentage by echocardiography in 10- to 12 -week-old mice showed no differences between CFKO and control mice (Table 1). Hemodynamic analysis by cardiac catheterization revealed that the baseline of $\mathrm{LV}$ contractility (assessed by maximal $d P / d t$ ) and diastolic function (assessed by minimal $d P / d t$ ) were similar in CFKO and control mice (Table 2). Dobutamine stimulation increased the cardiac function in both CFKO and control mice; however, there were no significant difference between CFKO and control mice. Together, 
these results suggested that deletion of FAK in cardiomyocytes did not affect cardiac function.

Increased hypertrophy of CFKO mice upon stimulation by Ang II and transverse aortic constriction. As a number of studies have suggested that genetic mutations can cause hypertrophy and heart failure under challenged conditions, despite not affecting basal cardiac function $(29,37)$, we examined the potential effect of cardiacspecific FAK inactivation on the hypertrophy induced by Ang II. Echocardiography was performed on the CFKO and control littermate mice 2 weeks after chronic administration of Ang II using implanted osmotic minipumps. As shown in Table 1, the LV chamber dimension was increased significantly during both systole and diastole in CFKO mice compared with the control littermates. No significant differences were detected for the thickness of interventricular septum, LV free wall, or fractional shortening between the $\mathrm{CFKO}$ and control mice. In addition to the chamber dilation evidenced by echocardiography, the ratios of heart to body weight and heart weight/tibial length were significantly increased in the CFKO mice compared with the control littermates (Figure 2, A and $\mathrm{C}$ ), whereas the ratio of liver to body weights was similar in all 3 groups of mice (Figure 2E). The chamber dilation with the increase in heart weight is consistent with eccentric hypertrophy in the CFKO mice upon Ang II stimulation.

Previous studies have shown that FAK plays an important role in transducing mechanical stress that leads to cardiomyocyte hypertrophy in vitro $(18,20,21)$. To investigate the role of FAK in biomechanical stress in cardiac in vivo, we performed transverse aortic constriction (TAC) on 8- to 10-week-old CFKO and control mice. The cardiac morphology and function were examined by echocardiography at 10 days after of surgery. Consistent with Ang II stimulation results, the LV chamber dimension induced by pressure overload was increased significantly during both systole and diastole in CFKO mice compared with the control littermates (Table 1). The heart/body weight and heart weight/tibial length ratios were also increased significantly in CFKO mice (Figure 2, B and $\mathrm{D}$ ), whereas the ratio of liver to body weight was similar in all 3 groups of mice (Figure 2F).

A number of reexpressed fetal cardiac genes, including $\alpha$-skeletal actin (SK-actin) and atrial natriuretic peptide (ANP), have been considered as markers for cardiac hypertrophy (38). We examined the expression of several cardiac hypertrophy markers in CFKO and control mice after Ang II (or vehicle alone) stimulation by Northern blot analysis. At 12 weeks of age, the basal level of SK-actin was increased in CFKO mice compared with control mice (Figure 3), despite no changes in the ratio of heart to body weight or echocardiography indexes (see Table 1). In Ang II-treated mice, ANP, brain natriuretic peptide (BNP), myosin heavy chain $\beta$ ( $\beta$-MHC), and SK-actin expression levels were induced to a higher level in CFKO mice compared with control mice. Expression of $\alpha-\mathrm{MHC}$ and sarcoplasmic reticulum $\mathrm{Ca}^{2+}$ ATPase (SERCA2a) were unchanged under all tested conditions. These results indicate that FAK deletion in ventricular cardiomyocytes increases the expression of cardiac hypertrophy markers ANP, BNP, $\beta$-MHC, and SK-actin and results in augmented cardiac hypertrophy upon Ang II stimulation in young mice.

Histological analysis reveals cardiac fibrosis in the CFKO mice stimulated with Ang II. To further investigate the potential differences in CFKO and control mice upon Ang II stimulation, hearts were dissected, fixed, and subjected to histological analysis. Microscope examination of sections stained with $\mathrm{H} \& \mathrm{E}$ did not reveal any significant 

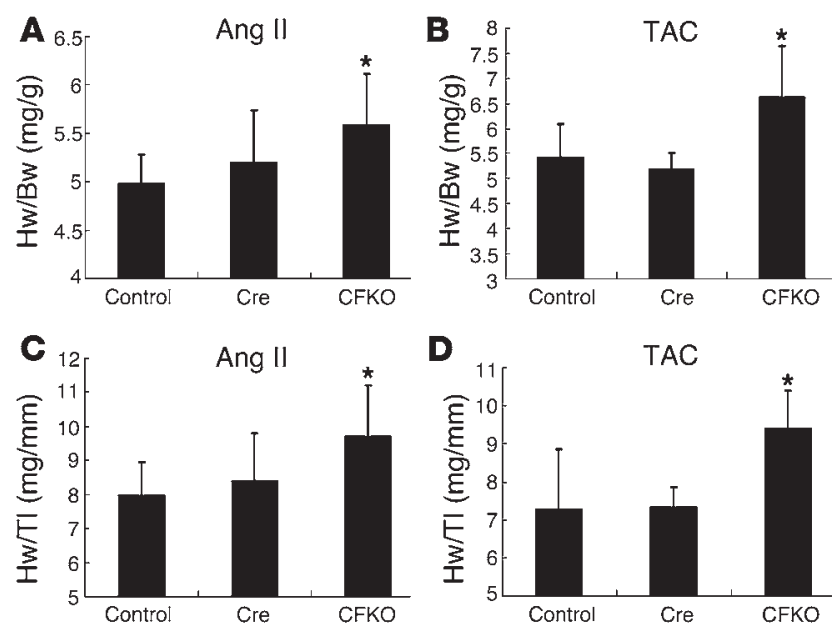

D
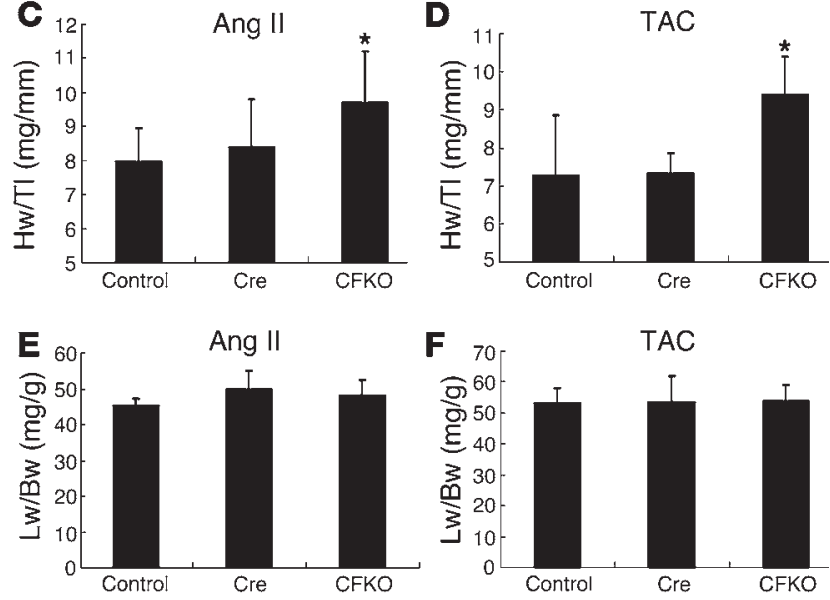

histological changes in CFKO mice compared with control littermates (Figure 4, A and B). To characterize myofiber cross-sectional area in more detail, histologic sections were stained with wheat germ agglutinin-TRITC (WGA-TRITC). The WGA-stained sections indicated that myofiber cross-sectional area was comparable between CFKO and control mice, suggesting that FAK deletion in the heart has minimal influence on the thickness of cardiomyocytes (data not shown). Masson trichrome staining revealed multifocal interstitial fibrosis in the myocardium of CFKO but not control mice (Figure 4, C and D).

Interstitial fibrosis in CFKO mice could be due to decreased collagen degradation or increased collagen synthesis in response to tissue damage. As FAK regulates expression and activation of several MMPs in vitro (39-41), we examined MMP activities in the heart of CFKO and control mice upon Ang II stimulation using zymography. The activities of MMP2 and MMP9 were found to be similar between samples from CFKO and control mice (data not shown), suggesting that the collagen degradation due to MMPs was not the main reason of cardiac fibrosis in CFKO mice. Northern blot analysis for collagen I, IV, and VI was performed to determine potential differences in their synthesis. As shown in Figure 4E, stimulation with Ang II increased the expression of collagen I, IV, and VI. Furthermore, the induced levels of collagens I and VI

Figure 3

Northern blot analysis of hypertrophy markers in CFKO mice. (A) The hearts of CFKO and control mice were harvested at 14 days after infusions with vehicle or Ang II, as indicated. The total ventricular RNA was isolated and subjected to Northern blotting with various probes, as indicated. (B) The intensity of the bands was quantified from 3 independent experiments by densitometry. The mean \pm SD values of relative intensity (normalized to the control sample treated with vehicle) are shown in arbitrary units. ${ }^{\star} P<0.05$ compared with values from control mice with Ang II infusion.

\section{Figure 2}

Cardiac hypertrophic responses upon angiotensin II stimulation in CFKO mice. Heart and liver were harvested at 14 days after Ang II infusion (A, $\mathbf{C}$, and $\mathbf{E})$ or 10 days after TAC (B, D, and $\mathbf{F})$, as described in Methods. Heart/body weight (Hw/Bw; mg/g) (A and B), heart weight/ tibial length $(\mathrm{Hw} / \mathrm{Tl} ; \mathrm{mg} / \mathrm{mm})(\mathbf{C}$ and $\mathbf{D})$, and liver/body weight ( $\mathrm{Lw} / \mathrm{Bw}$; $\mathrm{mg} / \mathrm{g})(\mathbf{E}$ and $\mathbf{F})$ ratios of the CFKO mice and their littermate floxed $\left(F A K^{f l o x / f l o x}\right)$ and Cre $\left(F A K^{f l o x /+} M L C 2 \mathrm{VCre}^{\mathrm{Kl} /+}\right)$ controls were determined. Heart and liver of at least 8 mice of each group were weighed, and values represent mean $\pm \mathrm{SD}$. ${ }^{*} P<0.05$ compared with values from floxed and Cre controls.

were higher in CFKO mice than the control mice, whereas the level of collagen IV was similar in CFKO and control mice (Figure 4F). These results suggest that increased collagen I and VI synthesis may be responsible for the multifocal interstitial fibrosis observed in CFKO mice upon Ang II stimulation.

FAK has been shown to promote cell survival by protection from apoptosis in several previous studies $(13,14,42)$. To determine whether eccentric cardiac hypertrophy and fibrosis are associated with increased cardiomyocyte apoptosis in CFKO mice, we performed TUNEL assays on heart sections from CFKO and control mice stimulated with Ang II. Apoptotic cells were detected in CFKO and control mice, and the fraction of apoptotic versus total cells was comparable in these mice (data not shown). This suggested that increased cardiac fibrosis and hypertrophy in the CFKO mice is not caused by increased cardiomyocyte apoptosis.

Spontaneous LV chamber dilation in old CFKO mice. The tolerance to multiple stresses is diminished with aging, and an impaired tolerance is also observed in isolated hearts (43). To test whether FAK gene deletion influences heart morphology and function in old mice, we performed echocardiography to examine CFKO and control mice at 9 months of age. A significant elevation of LV cham-

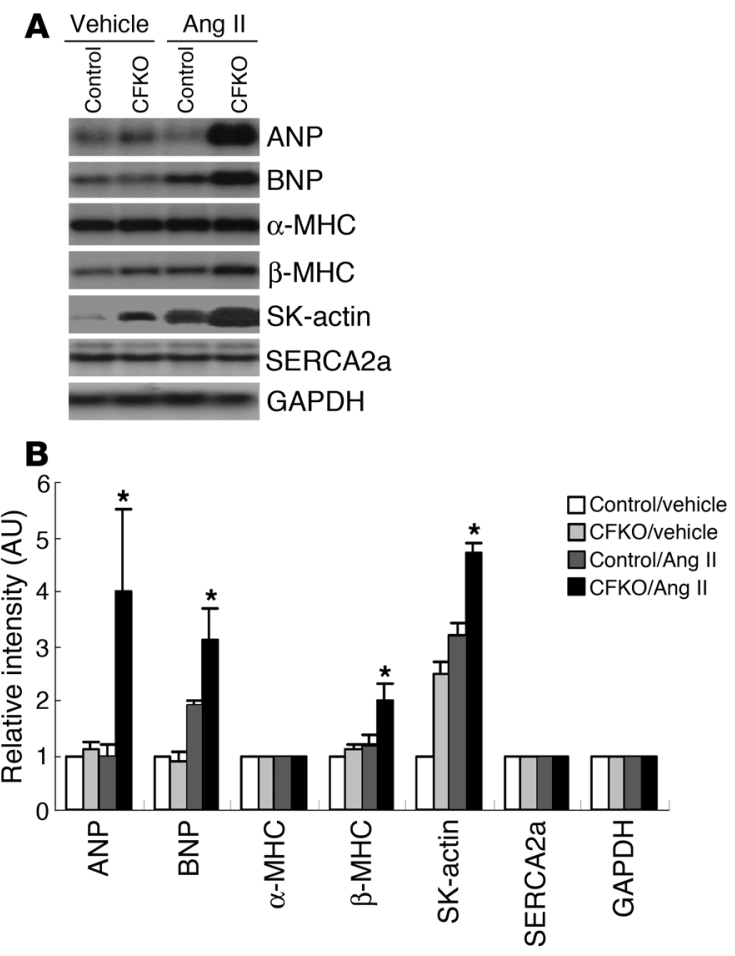



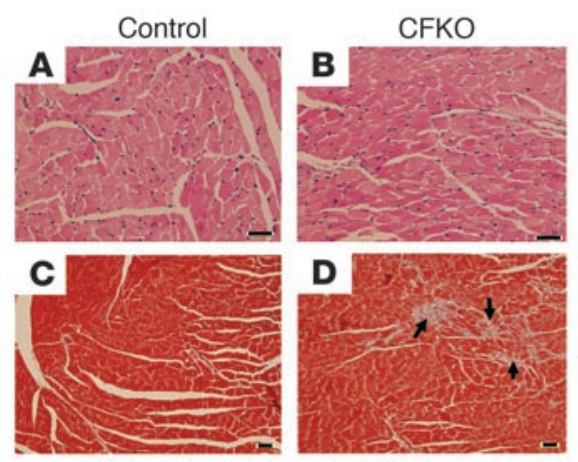

$H \& E$

Masson

trichrome
E

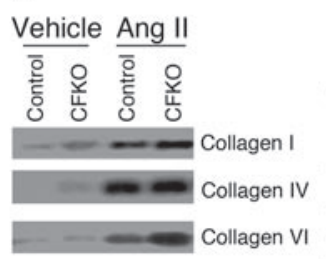

$\mathbf{F}$

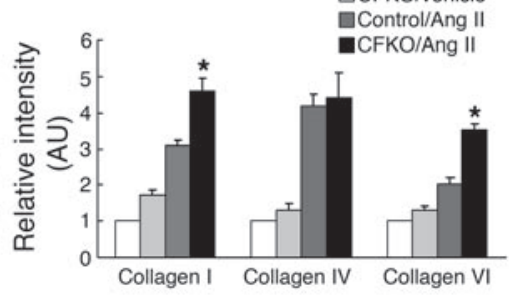

ber dimension was observed in both systole and diastole in CFKO mice compared with control mice (Table 1). Northern blot analysis of the heart in CFKO and control mice showed an increase in the expression of cardiac hypertrophy markers ANP, BNP, $\beta$-MHC, and SK-actin in CFKO mice compared with control mice (Figure 5 , A and B). These results suggested that deletion of the FAK gene in ventricular cardiomyocytes could cause spontaneous cardiac dilation in older mice.

To examine potential causes of cardiac chamber dilation, cardiomyocytes were isolated from 9-month-old CFKO and control mice.

\section{Figure 4}

Multifocal interstitial fibrosis of CFKO mice in response to Ang II infusion. (A-D) Cardiac sections from Ang II-infused control ( $\mathbf{A}$ and $\mathbf{C}$ ) or CFKO (B and D) mice were stained with H\&E (A and $\mathbf{B}$ ) or Masson trichrome (C and $\mathbf{D})$, as indicated. The interstitial fibrosis in sections from CFKO mice is marked by arrows. Scale bars: $60 \mu \mathrm{m}$. (E) Northern blot analysis for collagen I, IV, and VI expression of control or CFKO mice at 14 days after vehicle or Ang II infusion, as indicated. (F) The intensity of the bands was quantified from 3 independent experiments by densitometry. The mean \pm SD values of relative intensity (normalized to the control sample treated with vehicle) are shown in arbitrary units. ${ }^{*} P<0.05$ compared with values from control mice with Ang II infusion.

Morphometric analysis of the cells showed that cardiomyocytes from CFKO mice constituted a greater area than those from control mice (Figure 5C). This was due to an increase in the length, but not the width, of the cardiomyocytes from CFKO mice (Figure 5D). These results suggested that cardiac dilation may be a consequence of impaired myocyte length remodeling in older CFKO mice.

FAK-deficient cardiomyocytes have disorganized cytoarchitectural organization and defects in mitochondria. To further evaluate the abnormalities in the myocardium of CFKO mice, samples from ventricles of 9-month-old control and CFKO mice were examined by electron microscopy. We especially selected the field that revealed longitudinally oriented cardiomyocytes for evaluation. Control cardiomyocytes were well aligned and had organized myofibrils with intact $Z$ lines. The well-preserved mitochondria were located along the periphery of the longitudinally oriented cardiac myofibrils (Figure $6 \mathrm{~A})$. Note that the mitochondrial cristae are regularly spaced and horizontally oriented (Figure 6C). In contrast to the control mice, in the CFKO animals we observed disorganized myofibrils with a marked increase interfibrillar space containing large aggregates of swollen mitochondria (Figure 6B). The affected mitochondria

\section{Figure 5}

Cardiac hypertrophy in older CFKO mice. (A) The hearts of 9-month-old CFKO and control mice were harvested. The total ventricular RNA was isolated and subjected to Northern blotting with various probes, as indicated. (B) The intensity of the bands was quantified from 3 independent experiments by densitometry. The mean \pm SD values of relative intensity (normalized to the control mice) are shown in arbitrary units. ${ }^{*} P<0.05$ compared with values from control mice. (C and $\mathbf{D})$ Cardiomyocytes were isolated from CFKO and control mice as described in Methods. They were then analyzed for area (C) and for length of long and short axes (D) using Image-Pro Plus version 3.0 morphometric analysis software. The mean $\pm S D$ values from 3 independent experiments are shown. ${ }^{*} P<0.05$ compared with values from control mice. Representative images of isolated cardiomyocytes are also shown in $\mathbf{C}$.
A

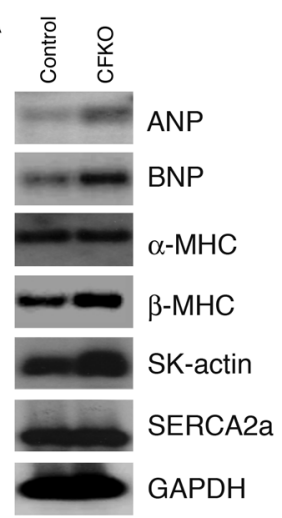

C

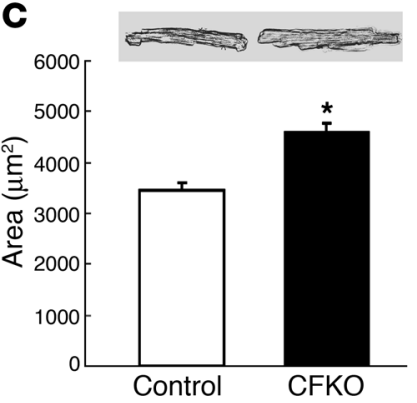

B
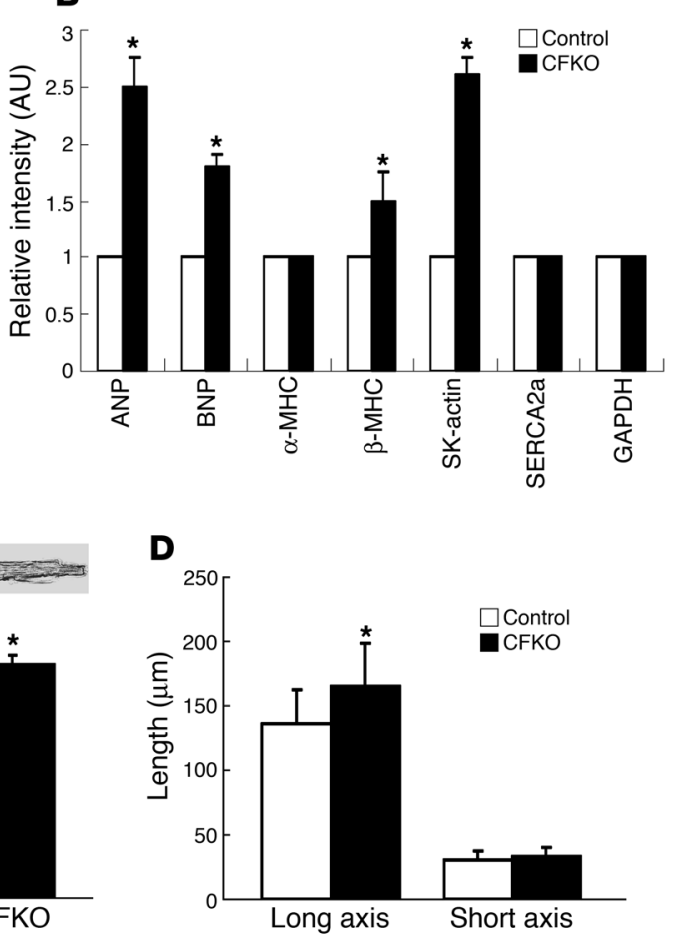

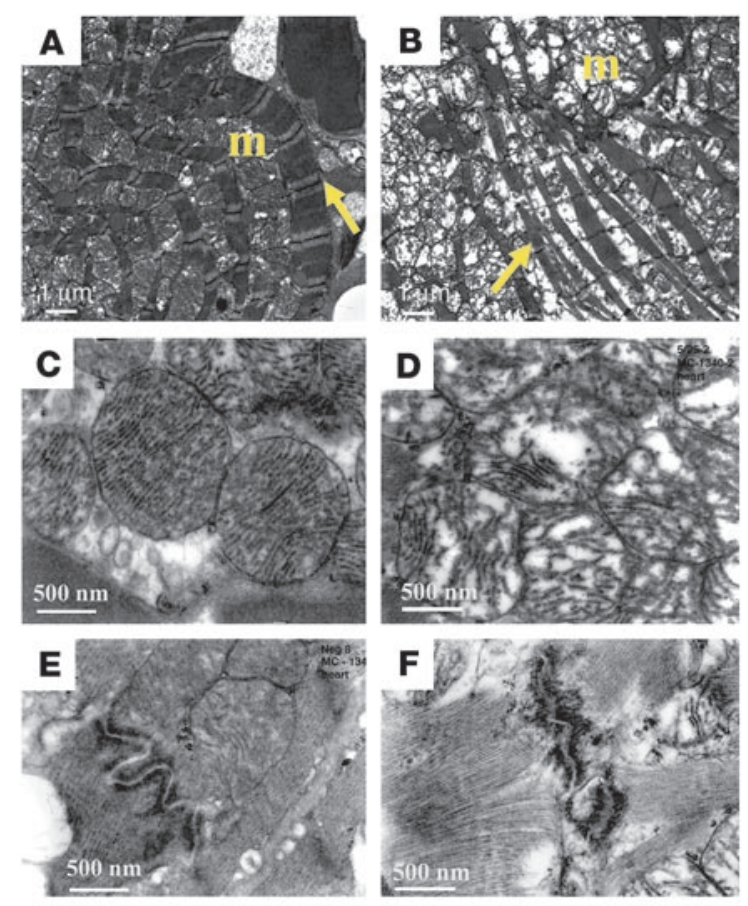

of CFKO mice showed irregular spread and disrupted cristae with partially lucent matrix (Figure 6D). The structure of $\mathrm{Z}$ disks in CFKO mice appeared normal compared with that in the control mice (data not shown). In contrast, while we found well-preserved intercalated disk with smooth contours in control cardiac section (Figure 6E), the disk region was straighter, with loss of the normal serpentine configuration and unusual sharp angles in CFKO mice. These results suggested a deranged cellular metabolism with abnormal cell-cell interactions upon FAK inactivation, which may contribute to the cardiac chamber dilation in CFKO mice.

Reduced phosphorylation of $p 130$ Cas and paxillin in the heart of CFKO mice. Multiple targets and signaling pathways have been implicated in mediating regulation of cellular functions by FAK (13-17). In particular, FAK substrates p130Cas and paxillin have been implicated in the regulation of cardiomyocyte function in previous studies (44-46). To explore potential molecular mechanisms of cardiac defects in the CFKO mice, we examined the phosphorylation states of $\mathrm{p} 130 \mathrm{Cas}$ and paxillin in these mice with or without Ang II stimulation. As shown in Figure 7, p130Cas phosphorylation was decreased in CFKO mice compared with control mice before and after Ang II stimulation. Stimulation of Ang II increased p130Cas phosphorylation in CFKO and control mice,

\section{Figure 7}

Phosphorylation of p130Cas and paxillin was decreased in CFKO mice. Osmotic minipumps containing Ang II were implanted into control and CFKO mice as described in Methods. At the indicated times, mice were euthanized and hearts were dissected. Lysates from the dissected hearts were immunoprecipitated with anti-p130Cas antibody followed by Western blotting with anti-PY20 or with anti-p130Cas or analyzed directly by Western blotting with anti-pY118-paxillin (p-paxillin) or anti-paxillin, as indicated. All blots are representative of at least 3 independent experiments.

\section{Figure 6}

Ultrastructural morphology of cardiomyocytes and mitochondrial defects in 9-month-old CFKO mice. Representative transmission electron micrographs of ventricle specimens from control $(\mathbf{A}, \mathbf{C}$, and $\mathbf{E})$ and CFKO (B, D, and F) mice. Well-organized myofibrils (arrows) with mitochondria $(\mathrm{m})$ grouped along the periphery of longitudinally oriented fibers are found in control mice (A), whereas myofibrillar disarray as well as pronounced increases in nonmyofibrillar space filled with mitochondria arranged in aggregates disorganizing fibrils were evident in CFKO mice (B). Mitochondria have tightly packed, intact horizontally oriented cristae in control mice (C), while in CFKO mice, the mitochondria are swelled, the affected mitochondrial cristae are no longer regular and horizontal, and their matrix is partially lucent and irregularly spaced (D). Well-preserved intercalated disk with smooth contours in control mice (E) and affected straighter intercalated disk with unusual sharp angles in CFKO mice (F). Magnification, $\times 7,000$ (scale bars: $1 \mu \mathrm{m} ; \mathbf{A}$ and $\mathbf{B}$ ) and $\times 30,000$ (scale bars: $500 \mathrm{~nm}$; C-F).

although the increases were lower in CFKO mice. Phosphorylation of paxillin at Tyr118, a critical phosphorylation site for paxillin regulation of cell spreading and migration (47), was also reduced in CFKO mice compared with control mice, although we did not observe stimulation of paxillin phosphorylation at this site by Ang II. Together, these data suggest that the decreases in p130Cas and paxillin phosphorylation may be responsible for the eccentric cardiac hypertrophy observed in young CFKO mice upon Ang II stimulation and spontaneously in older CFKO mice.

\section{Discussion}

As a major mediator of signaling by integrins as well as other cell-surface receptors, FAK has been implicated in the regulation and function of cardiomyocytes by several in vitro studies $(21,23,24,42,44,48,49)$. In addition, defective heart development was observed in FAK total KO embryos $(25,26)$. However, the early embryonic lethality in the total-KO mice precluded in vivo analysis of potential roles of FAK in heart development and FAK function in adult mice. Using a Cre-loxP-based conditional $\mathrm{KO}$ approach that specifically deletes the FAK gene in ventricular cardiomyocytes, we found that inactivation of FAK expression in the heart promoted eccentric cardiac hypertrophy in young mice stimulated with Ang II or pressure overload by TAC and spontaneous LV chamber dilation in older mice. To our knowledge, this is the first report to demonstrate a role for FAK in the regulation of heart hypertrophy in vivo.

Total KO of the FAK gene causes early embryonic death at E8.5 with extensive defects in heart development $(25,26)$. In contrast, CFKO mice with specific deletion of FAK in cardiomyocytes showed normal embryonic viability, raising the possibility that

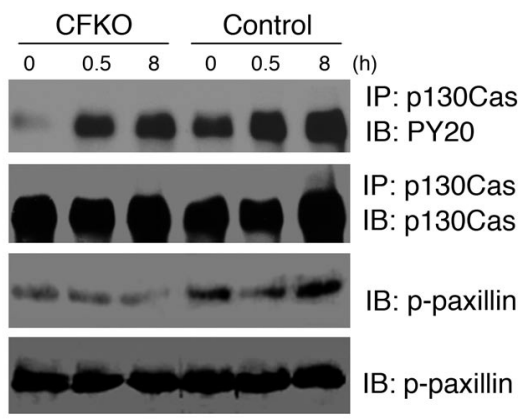


there is no cardiomyocyte cell-autonomous requirement for FAK in heart development. MLC2vCreKI is a well-established mouse line that has been shown to delete floxed genes specifically and efficiently in adult mouse heart in a number of previous studies $(12,28-34)$. However, the efficiency of deletion of floxed genes by MLC $2 \mathrm{vCreKI}$ in the embryos is less clear. Although previous reports have shown Cre-mediated deletion of floxed RXR $\alpha$ genes as early as E8.5 using this line (28), Crone et al. found recently that the ErbB2 protein level was not reduced at E18.5 in crosses between floxed ErbB2 and MLC2vCreKI mice, suggesting potentially inefficient cleavage of the floxed ErbB2 gene using the MLC2vCreKI mice (34). Indeed, our temporal analysis of FAK protein expression levels indicated that FAK expression was similar in cardiac ventricles of CFKO and control mice at E15.5 and postnatal day 0 , and a reduction of FAK protein was observed from 2 weeks till at least 40 weeks after birth (see Figure 1D). Therefore, it is likely that the presence of FAK in the embryonic heart is responsible for the apparent normal heart development in CFKO mice in contrast to the heart defects observed in the total FAK KO embryos.

We found that CFKO mice showed increased eccentric heart hypertrophy upon stimulation with Ang II and TAC and that older CFKO mice developed LV chamber dilation spontaneously. Mutations of many genes have been identified as causing different heart defects based on both human genetic studies and various mouse models. It is interesting that mutations in proteins of cytoskeletal structures are often associated with dilated cardiomyopathy characterized by ventricular chamber dilation, fibrosis, and heart failure (5). The cytoskeleton of myocytes includes the $\mathrm{Z}$ disk, which defines the lateral boundaries of the sarcomeres and is a key component of the sensor for cardiomyocyte stretch in vivo. The $\mathrm{Z}$ disk-associated complex of cardiomyocytes is analogous to focal adhesions in other cells, such as fibroblasts. Indeed, focal adhesion proteins, including integrins, vinculin, FAK, and p130Cas, have been localized in the $Z$ disk of cardiomyocytes (44, 45, 50-53). Recent in vitro studies showed that disruption of FAK localization to $Z$ disk perturbed the structure of the $Z$ disk (44). In addition, a recent study showed FAK localization to the intercalated disk in hypertrophied cardiomyocytes (54), suggesting a possible role of FAK in maintaining normal cell-cell interactions in intercalated disks. Analysis of myocardia of CFKO mice by electron microscopy showed disorganized myofibrils with increased nonmyofibrillar space filled by swelled and disorganized mitochondria in comparison to control mice. The affected intercalated disks in CFKO mice lost the normal serpentine configuration and had unusual sharp angles (Figure 6). Therefore, specific deletion of FAK in cardiomyocytes could disrupt or compromise cell-cell interaction in CFKO mice, which could contribute to myofibril disorganization and ventricular chamber dilation.

Recent studies using cultured neonatal rat cardiomyocytes (NRCMs) suggested that FAK activation plays a role in mediating cardiomyocyte hypertrophy in response to stimulation by mechanical stress or agonists for $\mathrm{G}$ protein-coupled receptors $(23,49)$. The correlation between cardiomyocyte hypertrophy and increased FAK phosphorylation suggests that FAK serves a prohypertrophic role in cultured NRCMs. This appears to be in conflict with our observation that deletion of FAK in cardiomyocytes resulted in an increased eccentric hypertrophy in vivo. Similar discrepancies have also been observed for overexpression of activated MKK3, MKK6, MEK5, JNK, and p38 when studied in the cultured NRCM models versus in the adult heart in vivo (55). One possible explanation for such discrepancies is that although the cultured NRCM model is well-established for the study of cardiomyocyte hypertrophy, it mimics more closely heart hypertrophy in developmental growth of the cardiomyocytes, rather than pathological hypertrophy in adult, like eccentric hypertrophy (55). Indeed, we found that the length of cardiomyocytes was increased in CFKO mice compared with that in controls, although the cross-sectional area as detected by staining with WGA-FITC was similar for CFKO and control mice. The increased length in cardiomyocytes from CFKO mice is consistent with a recent report by Mansour et al. showing that FAK was required for neonatal myocyte length remodeling following longitudinal stretch (56). These results suggested that cardiomyocyte elongation upon FAK inactivation may contribute to the eccentric hypertrophy in CFKO mice.

Fetal gene reexpression in adult heart is one of the characteristics of cardiac hypertrophy. Indeed, we found increased expression of several cardiac hypertrophy markers, ANP, BNP, $\beta$-MHC, and SK-actin, in both the Ang II-stimulated young mice and older mice without any stimulation that developed chamber dilation as evidenced by echocardiography. Interestingly, we also found that the level of SK-actin expression was increased in 12-week-old CFKO mice, even though echocardiographic indexes and ratio of heart weight to body weight remained unchanged. This suggested that fetal gene reexpression may be a more sensitive indicator for cardiac hypertrophy.

In conclusion, establishment of a cardiomyocyte-specific FAKdeletion mouse model allowed us to define a role for FAK in the regulation of cardiac hypertrophy in vivo. Interestingly, similar studies with heart-specific deletion of integrin $\beta 1$ also showed ventricular chamber dilation and fibrosis, which is consistent with the notion that FAK is a major mediator of integrin signaling, as suggested by numerous in vitro studies (13-17). However, it should be noted that the phenotype in CFKO mice was milder than that of the integrin $\beta 1$ conditional $\mathrm{KO}$ mice, suggesting involvement of proteins other than FAK in mediating integrin functions in the heart. For example, recent studies showed that deletion of melusin, a muscle-specific protein that interacts with the integrin $\beta 1$ cytoplasmic domain, led to dilated cardiomyopathy in response to pressure overload (57). It is also interesting to note that Ang II stimulation of CFKO mice, but not melusin-null mice, led to increased eccentric cardiac hypertrophy. This may be due to FAK participation in signaling by $G$ protein-coupled receptors in addition to its well-characterized role in integrin signaling $(23,49)$. It is hoped that the availability of the CFKO mice along with other mouse models will allow us to gain a better understanding of specific signaling pathways as well as their crosstalk in the regulation of heart development and functions in future studies.

\section{Methods}

Animal preparation. Generation of FAK-floxed mice has been described previously (27). FAK-floxed and $\mathrm{Cre}^{+}$double heterozygous $\left(\mathrm{FAK}^{\mathrm{flox} /+} \mathrm{Cre}^{+}\right.$) mice were generated by crossing FAK-floxed homozygous mice with MLC2vCreKI mice $(12,28-34)$. The genotypes of pups obtained by crossing $\mathrm{FAK}^{\mathrm{flox} /+} \mathrm{Cre}^{+}$mice with FAK-floxed homozygous mice were determined by PCR using the forward (AGGGCTGGTCTGCGCTGACAGG) and reverse (GCTGATGTCCCAAGCTATTCC) primers (Figure 1A). Cycling conditions were as follows: 3 cycles at $94^{\circ} \mathrm{C}$ for 3 minutes, $72^{\circ} \mathrm{C}$ for 2 minutes, and $67^{\circ} \mathrm{C}$ for 2 minutes; 30 cycles at $94^{\circ} \mathrm{C}$ for 1 minute, $72^{\circ} \mathrm{C}$ for 1 minute, and $67^{\circ} \mathrm{C}$ for 2 minutes; 1 cycle at $72^{\circ} \mathrm{C}$ for $10 \mathrm{~min}$ utes. The expected sizes of wild-type allele, floxed allele, and deleted allele were $1.5 \mathrm{~kb}, 1.6 \mathrm{~kb}$, and $550 \mathrm{bp}$, respectively. 
Mice were housed in a specific pathogen-free facility and handled in accordance with standard use protocols and animal welfare regulations. All study protocols were approved by the Institutional Animal Care and Use Committee of Cornell University and conform to the NIH Guide for the care and use of laboratory animals (publication number 85-23, revised 1996).

Southern blotting. Genomic DNA isolated from mouse lung and heart LV using phenol/chloroform was digested with SacI, electrophoresed on $0.7 \%$ agarose gels, and transferred to NYTRAN SuPerCharge nylon membrane (Schleicher \& Schuell BioScience Inc.) using the alkaline method (27). The probe for Southern blotting was located outside the short arm and produced by PCR using forward (TCCTTGGGTGGGTACAGAGAAAC) and reverse (GGGCAAGTGTAAAAGCAGAGGAG) primers. The probe was labeled with $\left[\alpha-{ }^{32} \mathrm{P}\right] \mathrm{dCTP}$ by a random primer DNA labeling kit (Invitrogen Corp.). The blots were then hybridized at $65^{\circ} \mathrm{C}$ using QuikHyb Hybridization Solution (Stratagene) according to the manufacturer's instructions and later exposed to Kodak film.

Isolation of adult mouse ventricular cardiomyocytes. Mouse ventricular cardiomyocytes were isolated from 12-week-old or 9-month-old mice using a modified collagenase digestion method as described previously (58-60). Mice were anesthetized by i.p. injection of sodium pentobarbital (100 $\mathrm{mg} / \mathrm{kg}$ ) and $100 \mathrm{U}$ of heparin. The heart was quickly removed from the chest, cannulated through the aorta, and perfused with perfusion buffer (113 mM NaCl, $4.7 \mathrm{mM} \mathrm{KCl}, 0.6 \mathrm{mM} \mathrm{KH}_{2} \mathrm{PO}_{4}, 1.2 \mathrm{mM} \mathrm{MgSO}_{4}, 12 \mathrm{mM}$ $\mathrm{NaHCO}_{3} 10 \mathrm{mM}, \mathrm{KHCO}_{3}, 10 \mathrm{mM}$ HEPES, $30 \mathrm{mM}$ taurine) for 3 minutes and then switched to perfusion buffer with collagenase type II $(0.3 \mathrm{mg} / \mathrm{ml}$; Worthington Biochemical Corp.) for 10-12 minutes. The ventricles were then cut into several chunks and incubated in the digestion solution for 10 minutes. $\mathrm{CaCl}_{2}$ was then added stepwise to give a final concentration of 1 $\mathrm{mM}$, and myocytes were plated on laminin-coated dishes for 1 hour. Cells were fixed with $4 \%$ paraformaldehyde for measurements of cell length and width. At least 100 cells were examined in each experiment using ImagePro Plus version 3.0 software (MediaCybernetics) or lysed to prepare cell lysates for Western blotting.

Western blotting and immunoprecipitation. The following antibodies were purchased as indicated: rabbit polyclonal FAK (C-20), p130Cas, and paxillin antibodies from Santa Cruz Biotechnology Inc.; rabbit polyclonal Pyk2, mouse mAb $\alpha$-phosphotyrosine PY20 from Transduction Laboratories (BD Biosciences - Pharmingen); rabbit polyclonal $\alpha$-phosphotyrosine paxillin (Tyr118), $\alpha$-phosphotyrosine PYK2 (Tyr402) from Cell Signaling Technology; rabbit polyclonal $\alpha$-phosphotyrosine FAK (Tyr397) from BioSource International; mouse $\mathrm{mAb} \alpha$-vinculin from Sigma-Aldrich. Hearts or lungs isolated from mice were washed 3 times with ice-cold PBS and then lysed with modified RIPA buffer $(50 \mathrm{mM}$ Tris$\mathrm{HCl}, \mathrm{pH} 7.5,150 \mathrm{mM} \mathrm{NaCl}, 1 \% \mathrm{NP}-40,1 \%$ sodium deoxycholate, $1 \mathrm{mM}$ sodium vanadate, $10 \mathrm{mM}$ sodium pyrophosphate, $10 \mathrm{mM} \mathrm{NaF}, 1 \%$ Triton $\mathrm{X}-100,0.5 \% \mathrm{SDS}, 0.1 \% \mathrm{EDTA}, 10 \mu \mathrm{g} / \mathrm{ml}$ leupeptin, $10 \mu \mathrm{g} / \mathrm{ml}$ aprotinin, and $1 \mathrm{mM}$ PMSF). Lysates were cleared by centrifugation, and total protein concentrations were determined using the Bio-Rad Protein Assay. Immunoprecipitation was carried out by incubating lysates with anti$\mathrm{p} 130 \mathrm{Cas}$ polyclonal antibody and protein $\mathrm{A}$ beads at $4^{\circ} \mathrm{C}$ overnight. The beads were then washed 5 times using PBS. After washing, the beads were boiled with loading buffer and resolved by SDS-PAGE. Western blotting was performed as described previously (61).

Murine cardiac bypertrophy model induced by Ang II infusion. To induce murine cardiac hypertrophy, ALZET osmotic minipumps (model 2002) that contained either Ang II $(432 \mu \mathrm{g} / \mathrm{kg} / \mathrm{d})$ or vehicle alone $(150 \mathrm{mM} \mathrm{NaCl}$ plus $0.01 \mathrm{~N}$ acetic acid) were implanted dorsally and subcutaneously in agematched (10-12 weeks) CFKO and control mice under sodium pentobarbital $(160 \mathrm{mg} / \mathrm{kg})$ anesthesia. Fourteen days after implantation of pumps, hearts and livers were harvested, and the heart weight/body weight $(\mathrm{mg} / \mathrm{g})$, liver weight/body weight $(\mathrm{mg} / \mathrm{g})$, and heart weight/tibial length $(\mathrm{mg} / \mathrm{mm})$ ratios were determined and compared in CFKO and control mice.

TAC surgery. CFKO and their littermate control mice (10-week-old) were anesthetized with sodium pentobarbital $(160 \mathrm{mg} / \mathrm{kg})$, and TAC was performed as described previously (62). Ten days after surgery, the internal diameter and wall thickness of LV were checked by echocardiography. Hearts and livers of sacrificed mice were dissected and weighed to compare heart weight/body weight $(\mathrm{mg} / \mathrm{g})$, liver weight/body weight $(\mathrm{mg} / \mathrm{g})$, and heart weight/tibial length ratios in $\mathrm{CFKO}$ and control mice.

Transthoracic echocardiography. Echocardiography was preformed on control and CFKO mice with or without Ang II stimulation. The mice were anesthetized with an i.p. injection of $160 \mathrm{mg} / \mathrm{kg}$ of sodium pentobarbital and the chest shaved. The mice were placed in a supine position on a Styrofoam ultrasound table, and a layer of ultrasonic gel was applied over the left hemithorax. Using a Vivid 7 ultrasound system (GE Medical Systems; General Electric Co.) equipped with a wide-band, multifrequency (5.8-14 $\mathrm{mHz}$ ) linear array probe (i13L), measurements were obtained from the M-mode display using 2-dimensional guidance. All images were acquired and interpreted using accepted criteria $(63,64)$ The Doppler gain was adjusted to obtain optimal clarity of the myocardium. Both sector width and depth were adjusted to obtain maximal frame rates (>100 frames/s) to achieve optimal resolution. The following measurements were obtained in both diastole and systole: interventricular septal wall thickness, LV free wall thickness, and LV internal diameter. Measurements were obtained from the short-axis imaging plane from 3 cardiac cycles and averaged. The echocardiographer was blinded to the animals' genotypes.

Cardiac catheterization. Cardiac catheterizations were performed as described previously $(65,66)$. Sex-matched, 8 -week-old mice were anesthetized with avertin $(375 \mathrm{mg} / \mathrm{kg}$ body wt, i.p.). Mice were laid naturally on a warm cushion $\left(37^{\circ} \mathrm{C}\right)$ and fixed with adhesive tape. A 1.4-F Millar catheter-tip micromanometer catheter was inserted through the right carotid artery into the LV, where pressure and volume were recorded. We monitored the position of the tip by the length of catheter advanced and observation of the pressure wave form. Hemodynamic measurements were recorded at baseline and following injection of dobutamine $(2.5 \mu \mathrm{g} /$ $\mathrm{kg}$ body wt). Analog inputs from the pressure transducer were amplified using an ARIA (Millar Instruments Inc.) amplifier and digitized with a data-acquisition system (Harvard Apparatus) at a rate of 1,000 Hz. All parameters were calculated from the average of 30 consecutive beats at the stabilized stage.

Hypertrophy marker analysis by Northern blotting. Total RNA was isolated from frozen murine heart with TRIzol (Invitrogen Corp.). RNA (15 $\mu \mathrm{g})$ was separated by $1 \%$ formaldehyde-agarose gel electrophoresis and transferred to a NYTRAN SuPerCharge nylon membrane (Schleicher $\&$ Schuell BioScience Inc.). ANP, BNP, $\alpha$-MHC, $\beta$-MHC, SK-actin, SERCA2a, and GAPDH probes were prepared by PCR as described previously (67) and labeled with $\left[\alpha^{-32} \mathrm{P}\right] \mathrm{dCTP}$ using a random primer DNA labeling kit (Invitrogen Corp.). Blots were hybridized at $65^{\circ} \mathrm{C}$ using QuikHyb Hybridization Solution (Stratagene).

Histological analysis. After euthanasia by $\mathrm{CO}_{2}$, mice were perfused from heart $\mathrm{LV}$ with fresh $4 \%$ paraformaldehyde ( $\mathrm{pH} 8.0$ ) and fixed with it overnight. After dehydration, samples were embedded in paraffin wax according to standard laboratory procedures. Sections of $4 \mu \mathrm{m}$ were stained with $\mathrm{H} \& \mathrm{E}$ for routine histopathological examination with light microscopy. Fibrosis was analyzed using Masson trichrome staining. The sarcolemmal membranes were stained with WGA-TRITC at $150 \mu \mathrm{g} / \mathrm{ml}$ to accurately identify heart myofiber diameter.

Transmission electronic microscopy. For electron microscope analysis, a small piece of myocardium was taken from the LV and immediately fixed in $2.5 \%$ glutaraldehyde in $0.1 \mathrm{M}$ sodium cacodylated buffer, $\mathrm{pH}$ 7.4. After fixation, 
the pieces were postfixed in $2 \%$ solution of osmium tetroxide and dehydrated in graded alcohol at $4{ }^{\circ} \mathrm{C}$. After resin embedding and polymerization, $1-\mu \mathrm{m}$-thick sections were initially cut. Ultrathin sections were stained with uranyl acetate and Reynolds lead citrate and observed with transmission electron microscopy at $80 \mathrm{kV}$ using a 201 Philips electron microscope. Electromicrographs were recorded on 4489 Kodak electron microscopy film. The negatives were scanned using a Nikon scanner, Scantouch 110, with the software Nikon Scan 5.3 software.

Statistics. Data are presented as mean \pm SD. Means were compared by 2-tailed Student's $t$ test or 1-way ANOVA when comparing multiple groups. $P \leq 0.05$ was considered statistically significant.

\section{Acknowledgments}

We are grateful to Mon-Li Chu of Thomas Jefferson University for cDNA probes for collagens I, IV, and VI. We thank our colleagues Tracy Stokol, Zara Melkoumian, Tamas Nagy, Boyi Gan, and Xiao- yang Wu for their critical reading of the manuscript and helpful comments. This research was supported by NIH grants HL73394 to J.-L. Guan and HL66100 to J. Chen. H. Gu is supported by an NIH Intramural Grant and the Irene Diamond Foundation.

Received for publication January 28, 2005, and accepted in revised form October 24, 2005.

Address correspondence to: Jun-Lin Guan, Department of Molecular Medicine, College of Veterinary Medicine, Cornell University, Ithaca, New York 14853, USA. Phone: (607) 253-3586; Fax: (607) 253-3708; E-mail: jg19@cornell.edu.

Tang-Long Shen's present address is: Department of Plant Pathology and Microbiology, National Taiwan University, Taipei, Republic of China.
1. Frey, N., and Olson, E.N. 2003. Cardiac hypertrophy: the good, the bad, and the ugly. Annu. Rev. Physiol. 65:45-79.

2. Molkentin, J.D., and Dorn, I.G., 2nd. 2001. Cytoplasmic signaling pathways that regulate cardiac hypertrophy. Annu. Rev. Physiol. 63:391-426.

3. MacLellan, W.R., and Schneider, M.D. 2000. Genetic dissection of cardiac growth control pathways. Annu. Rev. Physiol. 62:289-319.

4. Hunter, J.J., and Chien, K.R. 1999. Signaling pathways for cardiac hypertrophy and failure. N. Engl.J. Med. 341:1276-1283.

5. Chien, K.R. 1999. Stress pathways and heart failure. Cell. 98:555-558.

6. Katz, A.M. 2002. Maladaptive growth in the failing heart: the cardiomyopathy of overload. Cardiovasc. Drugs Ther. 16:245-249.

7. Chien, K.R., and Olson, E.N. 2002. Converging pathways and principles in heart development and disease: CV@CSH. Cell. 110:153-162.

8. Knoll, R., Hoshijima, M., and Chien, K. 2003. Cardiac mechanotransduction and implications for heart disease. J. Mol. Med. 81:750-756.

9. Wang, N., Butler, J.P., and Ingber, D.E. 1993. Mechanotransduction across the cell surface and through the cytoskeleton. Science. 260:1124-1127.

10. Bloch, W., et al. 1997. Beta 1 integrin is essential for teratoma growth and angiogenesis. J. Cell Biol. 139:265-278.

11. Stephens, L.E., et al. 1995. Deletion of beta 1 integrins in mice results in inner cell mass failure and peri-implantation lethality. Genes Dev. 9:1883-1895.

12. Shai, S.Y., et al. 2002. Cardiac myocyte-specific excision of the beta1 integrin gene results in myocardial fibrosis and cardiac failure. Circ. Res. 90:458-464.

13. Parsons, J.T. 2003. Focal adhesion kinase: the first ten years. J. Cell Sci. 116:1409-1416.

14. Hanks, S.K., Ryzhova, L., Shin, N.Y., and Brabek, J. 2003. Focal adhesion kinase signaling activities and their implications in the control of cell survival and motility. Front. Biosci. 8:d982-d996.

15. Schaller, M.D. 2001. Biochemical signals and biological responses elicited by the focal adhesion kinase. Biochim. Biophys. Acta. 1540:1-21.

16. Schlaepfer, D.D., and Mitra, S.K. 2004. Multiple connections link FAK to cell motility and invasion. Curr. Opin. Genet. Dev. 14:92-101.

17. Abbi, S., and Guan, J.L. 2002. Focal adhesion kinase: protein interactions and cellular functions. Histol. Histopathol. 17:1163-1171.

18. Franchini, K.G., Torsoni, A.S., Soares, P.H., and Saad, M.J. 2000. Early activation of the multicomponent signaling complex associated with focal adhesion kinase induced by pressure overload in the rat heart. Circ. Res. 87:558-565.
19. Kuppuswamy, D., et al. 1997. Association of tyrosine-phosphorylated c-Src with the cytoskeleton of hypertrophying myocardium. J. Biol. Chem. 272:4500-4508.

20. Laser, M., et al. 2000. Integrin activation and focal complex formation in cardiac hypertrophy. J. Biol. Chem. 275:35624-35630.

21. Seko, Y., Takahashi, N., Tobe, K., Kadowaki, T., and Yazaki, Y. 1999. Pulsatile stretch activates mitogenactivated protein kinase (MAPK) family members and focal adhesion kinase (p125(FAK)) in cultured rat cardiac myocytes. Biochem. Biophys. Res. Commun. 259:8-14.

22. Domingos, P.P., Fonseca, P.M., Nadruz, W., Jr., and Franchini, K.G. 2002. Load-induced focal adhesion kinase activation in the myocardium: role of stretch and contractile activity. Am. J. Physiol. Heart Circ. Physiol. 282:H556-H564.

23. Eble, D.M., et al. 2000. Endothelin-induced cardiac myocyte hypertrophy: role for focal adhesion kinase. Am. J. Physiol. Heart Circ. Physiol. 278:H1695-H1707.

24. Takahashi, N., et al. 1999. Vascular endothelial growth factor induces activation and subcellular translocation of focal adhesion kinase (p125FAK) in cultured rat cardiac myocytes. Circ. Res. 84:1194-1202.

25. Ilic, D., et al. 1995. Reduced cell motility and enhanced focal adhesion contact formation in cells from FAK-deficient mice. Nature. 377:539-544.

26. Furuta, Y., et al. 1995. Mesodermal defect in late phase of gastrulation by a targeted mutation of focal adhesion kinase, FAK. Oncogene. 11:1989-1995.

27. Shen, T.-L., et al. 2005. Conditional knockout of focal adhesion kinase in endothelial cells reveals its role in angiogenesis and vascular development in late embryogenesis. J. Cell Biol. 169:941-952.

28. Chen, J., Kubalak, S.W., and Chien, K.R. 1998. Ventricular muscle-restricted targeting of the RXRalpha gene reveals a non-cell-autonomous requirement in cardiac chamber morphogenesis. Development. 125:1943-1949.

29. Hirota, H., et al. 1999. Loss of a gp130 cardiac muscle cell survival pathway is a critical event in the onset of heart failure during biomechanical stress. Cell. 97:189-198.

30. Chen, J., et al. 1998. Selective requirement of myosin light chain $2 \mathrm{v}$ in embryonic heart function. J. Biol. Chem. 273:1252-1256.

31. Ding, J.H., et al. 2004. Dilated cardiomyopathy caused by tissue-specific ablation of SC35 in the heart. EMBO J. 23:885-896.

32. Smith, R.M., et al. 2004. Genetic depletion of cardiac myocyte STAT-3 abolishes classical preconditioning. Cardiovasc. Res. 63:611-616.

33. Pashmforoush, M., et al. 2004. Nkx2-5 pathways and congenital heart disease:loss of ventricular myocyte lineage specification leads to progressive cardiomyopathy and complete heart block. Cell. 117:373-386.

34. Crone, S.A., et al. 2002. ErbB2 is essential in the prevention of dilated cardiomyopathy. Nat. Med. 8:459-465.

35. O’Brien, T.X., Lee, K.J., and Chien, K.R. 1993. Positional specification of ventricular myosin light chain 2 expression in the primitive murine heart tube. Proc. Natl. Acad. Sci. U. S. A. 90:5157-5161.

36. Soonpaa, M.H., Kim, K.K., Pajak, L., Franklin, M., and Field, L.J. 1996. Cardiomyocyte DNA synthesis and binucleation during murine development. Am. J. Physiol. 271:H2183-H2189.

37. Badorff, C., et al. 2002. Fas receptor signaling inhibits glycogen synthase kinase 3 beta and induces cardiac hypertrophy following pressure overload. J. Clin. Invest. 109:373-381. doi:10.1172/ JCI200213779.

38. Schultz, J.E., et al. 1999. Fibroblast growth factor-2 mediates pressure-induced hypertrophic response. J. Clin. Invest. 104:709-719.

39. Hsia, D.A., et al. 2003. Differential regulation of cell motility and invasion by FAK. J. Cell Biol. 160:753-767.

40. Sein, T.T., et al. 2000. A role for FAK in the Concanavalin A-dependent secretion of matrix metalloproteinase-2 and -9. Oncogene. 19:5539-5542.

41. Hauck, C.R., Hsia, D.A., Puente, X.S., Cheresh, D.A., and Schlaepfer, D.D. 2002. FRNK blocks v-Srcstimulated invasion and experimental metastases without effects on cell motility or growth. EMBOJ. 21:6289-6302.

42. Heidkamp, M.C., Bayer, A.L., Kalina, J.A., Eble, D.M., and Samarel, A.M. 2002. GFP-FRNK disrupts focal adhesions and induces anoikis in neonatal rat ventricular myocytes. Circ. Res. 90:1282-1289.

43. Gonzalez, A.A., Kumar, R., Mulligan, J.D., Davis, A.J., and Saupe, K.W. 2004. Effects of aging on cardiac and skeletal muscle AMPK activity: basal activity, allosteric activation, and response to in vivo hypoxemia in mice. Am. J. Physiol. Regul. Integr. Comp. Physiol. 287:R1270-R1275.

44. Kovacic-Milivojevic, B., et al. 2001. Focal adhesion kinase and p130Cas mediate both sarcomeric organization and activation of genes associated with cardiac myocyte hypertrophy. Mol. Biol. Cell. 12:2290-2307.

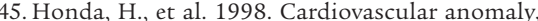
impaired actin bundling and resistance to Srcinduced transformation in mice lacking p130Cas. Nat. Genet. 19:361-365.

46. Melendez, J., et al. 2004. Cardiomyocyte apoptosis triggered by RAFTK/pyk2 via Src kinase is antagonized by paxillin. J. Biol. Chem. 279:53516-53523. 
47. Petit, V., et al. 2000. Phosphorylation of tyrosine residues 31 and 118 on paxillin regulates cell migration through an association with CRK in NBT-II cells. J. Cell Biol. 148:957-970.

48. Torsoni, A.S., Constancio, S.S., Nadruz, W., Jr., Hanks, S.K., and Franchini, K.G. 2003. Focal adhesion kinase is activated and mediates the early hypertrophic response to stretch in cardiac myocytes. Circ. Res. 93:140-147.

49. Taylor, J.M., Rovin, J.D., and Parsons, J.T. 2000. A role for focal adhesion kinase in phenylephrineinduced hypertrophy of rat ventricular cardiomyocytes. J. Biol. Chem. 275:19250-19257.

50. Zemljic-Harpf, A.E., et al. 2004. Heterozygous inactivation of the vinculin gene predisposes to stress-induced cardiomyopathy. Am. J. Pathol. 165:1033-1044.

51. Xu, W., Baribault, H., and Adamson, E.D. 1998. Vinculin knockout results in heart and brain defects during embryonic development. Development. 125:327-337.

52. Borg, T.K., et al. 2000. Specialization at the $Z$ line of cardiac myocytes. Cardiovasc. Res. 46:277-285.

53. Ross, R.S., and Borg, T.K. 2001. Integrins and the myocardium. Circ. Res. 88:1112-1119.

54. Yi, X.P., Wang, X., Gerdes, A.M., and Li, F. 2003. Subcellular redistribution of focal adhesion kinase and its related nonkinase in hypertrophic myocardium. Hypertension. 41:1317-1323.

55. Liang, Q., and Molkentin, J.D. 2003. Redefining the roles of $\mathrm{p} 38$ and JNK signaling in cardiac hypertrophy: dichotomy between cultured myocytes and animal models. J. Mol. Cell. Cardiol. 35:1385-1394.

56. Mansour, H., de Tombe, P.P., Samarel, A.M., and Russell, B. 2004. Restoration of resting sarcomere length after uniaxial static strain is regulated by protein kinase Cepsilon and focal adhesion kinase. Circ. Res. 94:642-649.

57. Brancaccio, M., et al. 2003. Melusin, a muscle-specific integrin beta1-interacting protein, is required to prevent cardiac failure in response to chronic pressure overload. Nat. Med. 9:68-75.

58. Zhou, Y.Y., et al. 2000. Culture and adenoviral infection of adult mouse cardiac myocytes: methods for cellular genetic physiology. Am. J. Physiol. Heart Circ. Physiol. 279:H429-H436.

59. Wolska, B.M., and Solaro, R.J. 1996. Method for isolation of adult mouse cardiac myocytes for studies of contraction and microfluorimetry. $\mathrm{Am}$. J. Physiol. 271:H1250-H1255.

60. Xin, H.B., et al. 2002. Oestrogen protects FKBP12.6 null mice from cardiac hypertrophy. Nature. 416:334-338.

61. Peng, X., et al. 2004. Overexpression of focal adhe- sion kinase in vascular endothelial cells promotes angiogenesis in transgenic mice. Cardiovasc. Res. 64:421-430.

62. Rockman, H.A., et al. 1991. Segregation of atrialspecific and inducible expression of an atrial natriuretic factor transgene in an in vivo murine model of cardiac hypertrophy. Proc. Natl. Acad. Sci. U. S. A. 88:8277-8281.

63. Sahn, D.J., DeMaria, A., Kisslo, J., and Weyman, A. 1978. Recommendations regarding quantitation in M-mode echocardiography: results of a survey of echocardiographic measurements. Circulation. 58:1072-1083.

64. Tanaka, N., et al. 1996. Transthoracic echocardiography in models of cardiac disease in the mouse. Circulation. 94:1109-1117.

65. Rogers, J.H., et al. 1999. RGS4 causes increased mortality and reduced cardiac hypertrophy in response to pressure overload. J. Clin. Invest. 104:567-576.

66. Nemoto, S., DeFreitas, G., Mann, D.L., and Carabello, B.A. 2002. Effects of changes in left ventricular contractility on indexes of contractility in mice. Am. J. Physiol. Heart Circ. Physiol. 283:H2504-H2510.

67. Wettschureck, N., et al. 2001. Absence of pressure overload induced myocardial hypertrophy after conditional inactivation of Galphaq/Galpha11 in cardiomyocytes. Nat. Med. 7:1236-1240. 